\title{
Many Tumor Infiltrating Lymphocytes
}

National Cancer Institute

\section{Source}

National Cancer Institute. Many Tumor Infiltrating Lymphocytes. NCI Thesaurus. Code C160235.

A finding of many tumor infiltrating lymphocytes in a biospecimen. 